\title{
Dynamic AdS/QCD and the spectrum of walking gauge theories
}

\author{
Timo Alho, ${ }^{1,2, *}$ Nick Evans, ${ }^{3, \dagger}$ and Kimmo Tuominen ${ }^{1,2, \sharp}$ \\ ${ }^{1}$ Department of Physics, University of Jyväskylä, P.O. Box 35, FIN-40014 Jyväskylä, Finland \\ ${ }^{2}$ Helsinki Institute of Physics, University of Helsinki, P.O. Box 64, FIN-00014 Helsinki, Finland \\ ${ }^{3}$ STAG Research Centre \& Physics and Astronomy, University of Southampton, Southampton SO17 1BJ, United Kingdom
}

(Received 6 September 2013; published 14 November 2013)

\begin{abstract}
We present a simple AdS/QCD model in which the formation of the chiral condensate is dynamically determined. The gauge dynamics is input through the running of the quark bilinear's anomalous dimension, $\gamma$. The condensate provides a dynamically generated infrared wall in the computation of mesonic bound state masses and decay constants. As an example, we use the model, with perturbative computations of the running of $\gamma$, to study $\mathrm{SU}(3)$ gauge theory with a continuous number of quark flavors, $N_{f}$. We follow the behavior of the spectrum as we approach the conformal window through a walking gauge theory regime. We show such walking theories display a Berezinskii-Kosterlitz-Thoules phase transition, with Miransky scaling, as one approaches the edge of the conformal window at the critical value of $N_{f}$. We show that these walking theories possess an enhanced quark condensate, a light Higgs-like excitation, and argue that the nonperturbative contribution to $\mathrm{S}$ falls to zero. We also study the deformation of the Berezinskii-Kosterlitz-Thoules transition when the quarks have a current mass, which may be of use for understanding lattice simulations of walking theories.
\end{abstract}

DOI: 10.1103/PhysRevD.88.105016

PACS numbers: $12.10 . \mathrm{Dm}, 11.25 . \mathrm{Tq}$

\section{INTRODUCTION}

The AdS/QCD models [1,2] provide a phenomenological holographic description of the QCD spectrum $(\pi \mathrm{s}, \rho \mathrm{s}$, and $a$ s) and are able to capture some of the elements of the theory. The simplest model, though, does not describe the dynamics of the generation of the quark condensate, instead putting it in by hand. The decoupling of the deep IR from the meson physics is also enacted by hand through a hard IR wall. In this paper we present an extension of these models, which includes all of this dynamics in one step. Our model is not a holographic description of the full theory since one must include the gauge dynamics via an assumed form for the running of the anomalous dimension of the quark antiquark bilinear, $\gamma$. Given this input, the IR dynamical quark mass and the usual AdS/QCD spectrum are predictions. In addition the model includes the scalar meson (which in QCD is associated with the physical $f_{0}$ ).

The model we present can be viewed as a natural evolution of work on D3/probe-D7 models [3] of chiral symmetry breaking [4] using the AdS/CFT correspondence [5], which we briefly review in the Appendix. There has been work on trying to describe the full gauge dynamics of chiral symmetry breaking theories in this system $[4,6]$, but it remains very hard to find true supergravity solutions of sufficiently complex dynamics. A phenomenological approach in this setup is to impose running on various anti-de Sitter (AdS) fields (such as the dilaton, which represents the gauge coupling) without worrying about backreaction

\footnotetext{
*timo.s.alho@jyu.fi

†evans@soton.ac.uk

kimmo.i.tuominen@jyu.fi
}

on the geometry [7]. Recently, though, we have shown that these models reduce in the quenched quark sector to dialing the renormalization group flow of the quark antiquark bilinear operator [8]. In the model we present in this paper, we extract that element of the more complex models. One advantage of the link to the more rigorous string constructions is that the implementation of the IR wall in the model can be cleanly linked to the formation of the quark condensate.

In Ref. [9] we investigated the core dynamics of this model for the quark condensate and the scalar meson. Here we couple that dynamical "engine" to the AdS/QCD model to provide a wider description of the spectrum. The model predictions are dependent on the assumed form of $\gamma$, and therefore the power of the model is not so much for two or three flavor QCD but in allowing us to compare the behavior of the spectrum of a set of theories as the form of the running changes. Such an application is to the $N_{f}$ dependence of the spectrum in $\mathrm{SU}\left(N_{c}\right)$ QCD. The $N_{f}$ dependence of these theories is of great theoretical interest since such theories may include strongly coupled IR conformal plasmas and theories with mass gaps that behave rather differently from QCD. There is a growing body of work on this subject including Refs. [10-31]. Other instructive holographic descriptions of this physics can be found in Refs. [32-46].

For a theory with quarks in the fundamental representation asymptotic freedom sets in when $N_{f}<11 / 2 N_{c}$. Immediately below that point, at least at large $N_{c}$, the two loop beta function enforces a perturbative IR fixed point $[47,48]$. The fixed point behavior is expected to persist into the nonperturbative regime as $N_{f}$ is further reduced [10]. At some critical value of the number of 
flavors, $N_{f}^{c}$, the coupling is expected to be strong enough to trigger chiral symmetry breaking by the formation of a quark antiquark condensate. The critical value, $N_{f}^{c}$, i.e., the lower boundary of the conformal window, can be estimated in a variety of ways [10-31]. Different semianalytic methods typically yield the chiral transition to occur below $N_{f}^{c} \simeq 4 N_{c}$ for fundamental fermion flavors. The chiral phase transition at the lower edge of the conformal window may give way to a regime of walking dynamics directly below $N_{f}^{c}$ [49]. For walking theories, there is expected to be a long energy range in which the coupling barely runs before tripping through the critical coupling value in the deep IR. Such theories display a tuned gap between the value of the quark condensate and the pion decay constant $f_{\pi}$, since the quark bilinear has a significant anomalous dimension, $\gamma$, over a large running regime. They are of interest phenomenologically for technicolor models of electroweak symmetry breaking [50,51] because flavor changing neutral currents are suppressed in extended technicolor models, and flavor physics is decoupled from the electroweak scale [49]. The walking dynamics may also suppress the contributions of the techniquarks to the electroweak oblique corrections, in particular to the $\mathrm{S}$ parameter [52,53]. Walking theories may also possess a parametrically light bound state, a pseudo-Goldstone boson of the breaking of dilatation symmetry by the quark condensate [54-57]. It is believed that in walking theories the intrinsic scale falls toward zero exponentially with $N_{f}^{c}-N_{f}$ as the conformal window is approached from below (known as Miransky scaling [58] or a holographic Berezinskii-Kosterlitz-Thoules (BKT) transition [59]).

In this paper we will take our running from the two loop perturbative result for the $\mathrm{SU}(3)$ gauge theory with a continuous flavor parameter $N_{f}$ allowing us to see all of the above structure and match the spectrum to that observed in QCD. The two loop result serves as an ansatz for the form of the running in the theory and has been used widely for that purpose, but it can not be relied upon beyond qualitative features in the strong coupling regime. Our simple AdS model will display all characteristics of walking theories as we approach the conformal window. Holographically the quark condensate becomes nonzero when the scalar describing it in AdS suffers an instability-this occurs when its mass passes through the Breitenlohner-Freedman (BF) bound of $m^{2}=-4$ [60]. Using the usual AdS/CFT dictionary $\left[m^{2}=\Delta(\Delta-4)\right]$, this corresponds to the point $\gamma=1$. Our assumption is that, as one decreases the continuous variable $N_{f}$ within the conformal window, the IR value of the scalar mass smoothly interpolates through -4 . We show this leads to a continuous transition with BKT scaling. The spectrum, which as a whole falls to zero mass as one approaches the critical value, $N_{f}^{c}$, displays a relatively light sigma meson close to the critical value (which one might hope to link to the observed light Higgs mass
$[61,62])$. The mass splitting between the vector and axial vector mesons in the model is determined by the gauge coupling constant $\kappa$ in the five dimensional model, which is a free parameter. To fix it we make use of the observed splitting in QCD. For this reason we will study the $N_{c}=3$ theory and fix $\kappa$ at $N_{f}=2$. We will treat $N_{f}$ as a continuous parameter even at $N_{c}=3$ since the behavior of the fixed points in the two loop QCD $\beta$ function show the same broad features with $N_{f}$ at all $N_{c}$. We believe all the features we observe will be present at any value of $N_{c}$ as one approaches the transition to the conformal window-of course formally only at the Veneziano limit (i.e., taking both $N_{c}$ and $N_{f}$ infinite with $x_{f} \equiv N_{c} / N_{f}$ fixed) can one treat $N_{f}$ as a truly continuous parameter. As one moves to higher $N_{f}$, the dependence of $\kappa$ on $N_{f}$ is crucial-we assume that as we approach the continuous chiral symmetry restoration transition at $N_{f}^{c}$ the mass difference must fall to zero so that axial-vector symmetry is smoothly restored. Given this assumption it naturally follows that the contribution of vector and axial vector mesons to the $S$ parameter falls to zero at the symmetry restoration point.

Finally we study the effect of including an explicit quark mass so the quarks are blind to the deep IR of the theory. The result is a deformation of the BKT-type scaling. When the scale where the theory violates the BF bound is larger than the hard quark mass, the theory behaves as the massless theory. The dynamics then smoothly moves over, as the quark mass becomes larger than the dynamical scale, to a regime where all mass scales in the theory (bound state masses, decay constants, and so forth) scale as simple powers of the hard mass, as determined by dimensional analysis. These results will potentially be of use to guide lattice simulations of walking dynamics where the chiral limit is hard to achieve.

\section{DYNAMIC ADS/QCD}

The essential dynamics of our model is encoded into a field $X$ of mass dimension 1 . The modulus of this field describes the quark condensate degree of freedom. Fluctuations in $|X|$ around its vacuum configurations will describe the scalar meson. The $\pi$ fields are the phase of $X$

$$
X=L(\rho) e^{2 i \pi^{a} T^{a}} .
$$

Here $\rho$ is the holographic coordinate ( $\rho=0$ is the IR, $\rho \rightarrow$ $\infty$ is the UV, and $|X|=L$ enters into the effective radial coordinate in the space, i.e., $r^{2}=\rho^{2}+|X|^{2}$. This is how the quark condensate will generate a soft IR wall: when $L$ is nonzero the theory will exclude the deep IR at $r=0$. This implementation is taken directly from the D3/probeD7 model [3] where $L$ is the embedding of the D7-brane in the AdS spacetime. Fluctuations on the brane then see the pulled back metric on the D7 world volume. We briefly review the D3/D7 case in the Appendix. 
We work with the five dimensional metric

$$
d s^{2}=\frac{d \rho^{2}}{\left(\rho^{2}+|X|^{2}\right)}+\left(\rho^{2}+|X|^{2}\right) d x^{2},
$$

which will be used for contractions of the space-time indices. The five dimensional action of our effective holographic theory is

$$
\begin{aligned}
S= & \int d^{4} x d \rho \operatorname{Tr} \rho^{3}\left[\frac{1}{\rho^{2}+|X|^{2}}|D X|^{2}+\frac{\Delta m^{2}}{\rho^{2}}|X|^{2}\right. \\
& \left.+\frac{1}{2 \kappa^{2}}\left(F_{V}^{2}+F_{A}^{2}\right)\right],
\end{aligned}
$$

where $F_{V}$ and $F_{A}$ are vector fields that will describe the vector $(V)$ and axial $(A)$ mesons. Note that we have not written the $\sqrt{-g}$ factor in the metric as $r^{3}$ but just $\rho^{3}$. Again, this is driven by the $\mathrm{D} 7$ probe action in which this factor is $\rho^{3}$; maintaining this form is crucial to correctly implementing the soft wall behavior. Finally $\kappa$ is a constant that will determine the $V-A$ mass splitting; we will fix its value and $N_{f}$ dependence in our model below.

The model presented is phenomenological in nature and we have included the bare minimum of content to reproduce the broad physics we expect. Thus, for example, we include a mass term for $X$ so that we may encode the running of the anomalous dimension of the quark bilinear. Higher order terms in $X$ would presumably also be present but we have no handle to fix their form so we omit them. These terms would be expected to change the quantitative values for meson masses and other observables that we present below but the qualitative behaviours are expected to be more stable.

The normalizations are determined by matching to the gauge theory in the UV of the theory. External currents are associated with the non-normalizable modes of the fields in AdS. In the UV we expect $|X| \sim 0$, and we can solve the equations of motion for the scalar, $L=K_{S}(\rho) e^{-i q . x}$, vector $V^{\mu}=\epsilon^{\mu} K_{V}(\rho) e^{-i q \cdot x}$, and axial $A^{\mu}=\epsilon^{\mu} K_{A}(\rho) e^{-i q \cdot x}$ fields. Each satisfies the same equation:

$$
\partial_{\rho}\left[\rho^{2} \partial_{\rho} K\right]-\frac{q^{2}}{\rho} K=0 .
$$

The UV solution is

$$
K_{i}=N_{i}\left(1+\frac{q^{2}}{4 \rho^{2}} \ln \left(q^{2} / \rho^{2}\right)\right), \quad(i=S, V, A),
$$

where $N_{i}$ are normalization constants that are not fixed by the linearized equation of motion. Substituting these solutions back into the action gives the scalar correlator $\Pi_{S S}$, the vector correlator $\Pi_{V V}$, and axial vector correlator $\Pi_{A A}$. Performing the usual matching to the UV gauge theory requires us to set

$$
N_{S}^{2}=\frac{N_{c} N_{f}}{24 \pi^{2}}, \quad N_{V}^{2}=N_{A}^{2}=\frac{\kappa^{2} N_{c} N_{f}}{24 \pi^{2}} .
$$

These choices should be compared to those in Refs. [1,2]: In Ref. [2] the entire action is multiplied by the factor of $N_{S}^{2}$ so that the normalizations of the fields can be set to 1 . Then in Ref. [2] the choice $\kappa=1$ is imposed so the scalar and vector correlators look symmetric. In Ref. [1] $\kappa$ is taken to equal our choice of $1 / N_{S}^{2}$, making the vector and axial normalizations unity (but the scalar normalization is not 1). The key point here is that $\kappa$ is not determined by the matching we have performed.

In the next section, we will derive the equations for the vacuum profile of the scalar $X$, the equations of motion for the mesons, and the formulas for evaluating the decay constants subject to the normalization conditions on external currents we have just determined in the UV.

\section{A. Vacuum structure and fluctuations}

Let us first consider the vacuum structure of the theory by setting all fields except $|X|=L$ to zero. We further assume that $L$ will have no dependence on the $x$ coordinates. The action for $L$ is given by

$$
S=\int d^{4} x d \rho \rho^{3}\left[\left(\partial_{\rho} L\right)^{2}+\Delta m^{2} \frac{L^{2}}{\rho^{2}}\right] .
$$

Now if we rewrite $L=\rho \phi$ and integrate the first term by parts, we arrive at

$$
S=\int d^{4} x d \rho\left(\rho^{5}\left(\partial_{\rho} \phi\right)^{2}+\rho^{3}\left(-3+\Delta m^{2}\right) \phi^{2}\right),
$$

which is the form for a canonical scalar in $\mathrm{AdS}_{5}$. The usual AdS relation between the scalar mass squared and the dimension of the field theory operator applies [ $\left.m^{2}=\Delta(\Delta-4)\right]$. If $\Delta m^{2}=0$, then the scalar describes a dimension 3 operator and dimension 1 source as is required for it to represent $\bar{q} q$ and the quark mass $m$. That is, in the UV the solution for the $\phi$ equation of motion is $\phi=m / \rho+\bar{q} q / \rho^{3}$.

The Euler-Lagrange equation for the determination of $L$, in the case of a constant $\Delta m^{2}$, is

$$
\partial_{\rho}\left[\rho^{3} \partial_{\rho} L\right]-\rho \Delta m^{2} L=0 .
$$

We have introduced $\Delta m^{2}$ in the full Lagrangian of the model in the minimal way consistent with changing the mass squared in the linearized regime. We can now ansatz an $r$ dependent $\Delta m^{2}$ to describe the running of the dimension of $\bar{q} q$. If the mass squared of the scalar violates the BF bound of $-4\left(\Delta m^{2}=-1\right)$, then we expect the scalar field $L$ to become unstable and settle to some nonzero value. If $\Delta m^{2}$ depends on $L$, then there is an additional term $-\rho L^{2} m^{2 \prime}(L)$ in the above equation of motion. At the level of the equation of motion, this is an effective contribution to the running of the anomalous dimension $\gamma$ that depends on the gradient of the rate of running in the gauge theory. At one loop in the gauge theory, there is no such term, and so we will neglect this term, effectively imposing the renormalization group (RG) running of $\Delta m^{2}$ only at the 
level of the equations of motion. Of course it is entirely appropriate to drop the term when the rate of running is small, which will be the case near the edge of the conformal window in the model below. For this reason including this term or otherwise makes no effect on the behavior of the model as one approaches the phase transition from the chiral symmetry breaking phase to the conformal window, which is our main interest here.

The solution for $L(\rho)$ can be found numerically by shooting from $\rho=0$ with the IR boundary condition $L^{\prime}(0)=0$. Adjusting the value of $L(0)$, the effective IR quark mass, $m_{q}$, one can find the regular flow that has $L(\infty)=m_{q}$ to describe a particular current quark mass. Until our final section, we will set $m_{q}=0$ and study the chiral limit. The first two terms generate the dynamics with $L$ becoming unstable if $\Delta m^{2}<-1$ over some range of $\rho$.

We next compute the scalar $\bar{q} q$ meson masses of our model. We look for space-time dependent excitations on top of the vacuum configuration, $L_{0}$, i.e., $|X|=L_{0}+$ $\delta(\rho) e^{-i q \cdot x}, q^{2}=-M^{2}$. The equation of motion for $\delta$ is, linearizing Eq. (9),

$$
\begin{gathered}
\partial_{\rho}\left(\rho^{3} \delta^{\prime}\right)-\Delta m^{2} \rho \delta-\left.\rho L_{0} \delta \frac{\partial \Delta m^{2}}{\partial L}\right|_{L_{0}} \\
+M^{2} R^{4} \frac{\rho^{3}}{\left(L_{0}^{2}+\rho^{2}\right)^{2}} \delta=0 .
\end{gathered}
$$

We seek solutions with, in the UV, asymptotics of $\delta=1 / \rho^{2}$ and with $\delta^{\prime}(0)=0$ in the IR, giving a discrete meson spectrum.

We must normalize $\delta$ so that the kinetic term of the $\sigma$ meson is canonical, i.e.,

$$
\int d \rho \frac{\rho^{3}}{\left(\rho^{2}+L^{2}\right)^{2}} \delta^{2}=1
$$

The scalar meson decay constant can be found using the solutions for the normalizable and non-normalizable wave functions. We concentrate on the action term (after integration by parts)

$$
S=\int d^{4} x d \rho \partial_{\rho}\left(-\rho^{3} \partial_{\rho} L\right) L .
$$

We substitute in the normalized solution $\delta$ and the external non-normalizable scalar function $K_{S}$ at $q^{2}=0$ with normalization $N_{S}$ to obtain the dimension 1 decay constant $f_{S}$ as

$$
f_{S}^{2}=\int d \rho \partial_{\rho}\left(-\rho^{3} \partial_{\rho} \delta\right) K_{S}\left(q^{2}=0\right) .
$$

The vector meson spectrum is determined from the normalizable solution of the equation of motion for the spatial pieces of the vector gauge field $V_{\mu \perp}=\epsilon^{\mu} V(\rho) e^{-i q . x}$ with $q^{2}=-M^{2}$. The appropriate equation is

$$
\partial_{\rho}\left[\rho^{3} \partial_{\rho} V\right]+\frac{\rho^{3} M^{2}}{\left(L_{0}^{2}+\rho^{2}\right)^{2}} V=0 .
$$

We again impose $V^{\prime}(0)=0$ in the IR and require in the UV that $V \sim c / \rho^{2}$. To fix $c$ we normalize the wave functions such that the vector meson kinetic term is canonical,

$$
\int d \rho \frac{\rho^{3}}{\kappa^{2}\left(\rho^{2}+L_{0}^{2}\right)^{2}} V^{2}=1
$$

The vector meson decay constant is given by substituting the solution back into the action and determining the coupling to an external $q^{2}=0$ vector current with wave function $K_{V}$. We have for the dimension $1 f_{V}$

$$
f_{V}^{2}=\int d \rho \frac{1}{\kappa^{2}} \partial_{\rho}\left[-\rho^{3} \partial_{\rho} V\right] K_{V}\left(q^{2}=0\right) .
$$

Note here that the factors of $\kappa$ cancel against those in the normalizations of $V, K_{V}$ and the result is $\kappa$ independent.

The axial meson spectrum is determined from the equation of motion for the spatial pieces of the axial vector gauge field. In the $A_{z}=0$ gauge, we write $A_{\mu}=A_{\mu \perp}+$ $\partial_{\mu} \phi$. The appropriate equation with $A_{\mu \perp}=\epsilon^{\mu} A(\rho) e^{-i q . x}$ with $q^{2}=-M^{2}$ is

$\partial_{\rho}\left[\rho^{3} \partial_{\rho} A\right]-\kappa^{2} \frac{L_{0}^{2} \rho^{3}}{\left(L_{0}^{2}+\rho^{2}\right)^{2}} A+\frac{\rho^{3} M^{2}}{\left(L_{0}^{2}+\rho^{2}\right)^{2}} A=0$.

The asymptotic behavior, boundary condition at $\rho=0$, and the normalization of $A(\rho)$ are same as those for $V(\rho)$. The axial meson decay constant is given by Eq. (16) with replacement $V \rightarrow A$.

The $\phi^{a}$ and $\pi^{a}$ equations are mixed and of the form

$$
\begin{gathered}
\partial_{\rho}\left[\rho^{3} \partial_{\rho} \phi^{a}\right]-\kappa^{2} \frac{\rho^{3} L^{2}}{\left(\rho^{2}+L^{2}\right)^{2}}\left(\pi^{a}-\phi^{a}\right)=0, \\
-q^{2} \partial_{\rho} \phi^{a}+\kappa^{2} L^{2} \partial_{\rho} \pi^{a}=0 .
\end{gathered}
$$

Note that there is always a solution of these equations where $q^{2}=0$ and $\phi^{a}=\pi^{a}=\mathrm{a}$ constant. The linearized field in the action associated with the pion is $X \sim 2 \pi i L_{0}$. Only in the case where the quark mass is zero and $L \sim$ $c / \rho^{2}$ asymptotically is this a normalizable fluctuation corresponding to a physical state in the field theory-it is the massless pion. When the quark mass is nonzero, this state is a flat direction of the theory only if one makes a spurious transformation on $\bar{q}_{L} q_{R}$ and $m$ simultaneously-i.e., it is not a physical state in the spectrum.

The pion decay constant can be extracted from the expectation that $\Pi_{A A}=f_{\pi}^{2}$. From the $f_{A}$ kinetic term with two external (non-normalizable) axial currents at $Q^{2}=0$, we obtain

$$
f_{\pi}^{2}=\int d \rho \frac{1}{\kappa^{2}} \partial_{\rho}\left[\rho^{3} \partial_{\rho} K_{A}\left(q^{2}=0\right)\right] K_{A}\left(q^{2}=0\right) .
$$




\section{TWO LOOP RUNNING INSPIRED MODEL}

To enact a realization of our model, we must choose how the anomalous dimension of $\langle\bar{q} q\rangle$ runs with the energy scale through the function $\Delta m^{2}$. We will choose to look at the $N_{f}$ dependence of $\mathrm{SU}\left(N_{c}\right)$ gauge dynamics to study the implications for the spectrum in the walking gauge theories expected to lie on the edge of the transition from the chiral symmetry breaking phase to the conformal window. We will use the perturbative running from $\mathrm{SU}\left(N_{c}\right)$ gauge theories with $N_{f}$ flavors since the two loop results display a conformal window.

The two loop running of the gauge coupling in QCD is given by

$$
\mu \frac{d \alpha}{d \mu}=-b_{0} \alpha^{2}-b_{1} \alpha^{3}
$$

where

$$
b_{0}=\frac{1}{6 \pi}\left(11 N_{c}-2 N_{F}\right) \text {, }
$$

and

$$
b_{1}=\frac{1}{24 \pi^{2}}\left(34 N_{c}^{2}-10 N_{c} N_{f}-3 \frac{N_{c}^{2}-1}{N_{c}} N_{F}\right) .
$$

Asymptotic freedom is present provided $N_{f}<11 / 2 N_{c}$. There is an IR fixed point with value

$$
\alpha_{*}=-b_{0} / b_{1},
$$

which rises to infinity at $N_{f} \sim 2.6 N_{c}$.

The one loop result for the anomalous dimension is

$$
\gamma=\frac{3 C_{2}}{2 \pi} \alpha=\frac{3\left(N_{c}^{2}-1\right)}{4 N_{c} \pi} \alpha .
$$

So, using the fixed point value $\alpha_{*}$, the condition $\gamma=1$ occurs at $N_{f}^{c} \sim 4 N_{c}$.

We will identify the RG scale $\mu$ with the AdS radial parameter $r=\sqrt{\rho^{2}+L^{2}}$ in our model. Note it is important that $L$ enters here. If it did not and the scalar mass were only a function of $\rho$, then were the mass to violate the BF bound at some $\rho$ it would leave the theory unstable however large $L$ grew. Including $L$ means that the creation of a nonzero but finite $L$ can remove the BF bound violation leading to a stable solution. Again in the D3/D7 system, this mechanism is very natural as discussed in the Appendix.

Working perturbatively from the AdS result $m^{2}=$ $\Delta(\Delta-4)$, we have

$$
\Delta m^{2}=-2 \gamma=-\frac{3\left(N_{c}^{2}-1\right)}{2 N_{c} \pi} \alpha .
$$

This will then fix the $r$ dependence of the scalar mass through $\Delta m^{2}$ as a function of $N_{c}$ and $N_{f}$. We sketch $\Delta m^{2}$ against $r$ for various cases in Fig. 1, showing the IR fixed

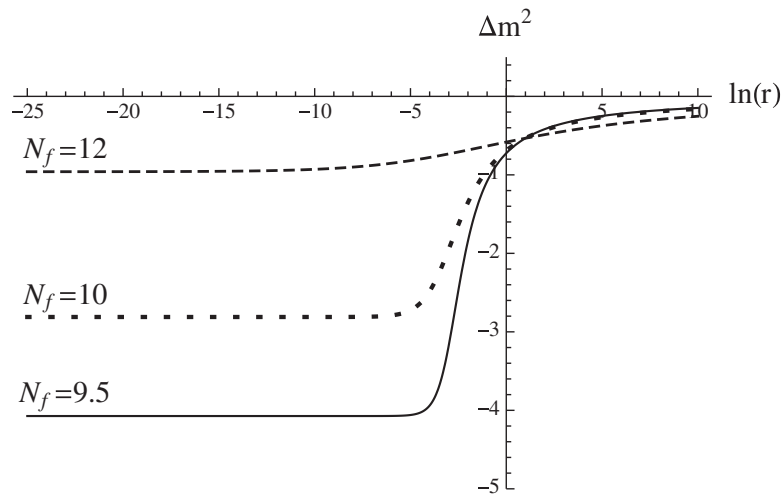

FIG. 1. $\Delta m^{2}$ vs $\ln (r)$ for $N_{c}=3, N_{f}=12,10,9.5$ showing the increasing magnitude of the IR fixed point with lowering $N_{f}$. Note that the BF bound is violated in the IR for $N_{f}<N_{f}^{c} \simeq 12$. Here we are comparing theories with the same value of $\alpha$ at $\ln (r)=1$.

point behavior. When solving numerically for the running coupling, we fix to the ultraviolet physics at scale $\ln \mu=1$ by setting $\alpha(\mu)=0.1$ across theories with different $N_{f}$.

To completely specify the model, we must also fix the parameter $\kappa$, which only enters into the computation of the axial meson mass and decay constant. It is natural therefore to try to fix the parameter to give the observed mass splitting of vector and axial vector mesons in QCD. This leads us to fix $N_{c}=3$ for our analysis here and vary $N_{f}$. Of course we are really just choosing the RG flow of $\gamma$, and using perturbative results for this when $\alpha$ becomes large is at best questionable. It is a model, and given this we will treat $N_{f}$ as a continuous parameter even at $N_{c}=3$ since then we will be able to smoothly move from a weakly coupled IR fixed point through to larger IR values and watch the behavior of the spectrum. The generic features we will see should be applicable at other values of $N_{c}$.

We fix $\kappa$ so that the correct values of vector and axial vector masses are reproduced at $N_{f}=2$. Then we must further choose how to make $\kappa$ scale with $N_{f}$. As discussed above we expect axial vector symmetry to be smoothly restored at the continuous chiral phase transition. We therefore require $\kappa \rightarrow 0$ at the transition at $N_{f}^{c}(\simeq 12)$. Taking all of these considerations into effect, we choose

$$
\kappa^{2}=3.34\left(N_{f}-N_{f}^{c}\right)
$$

Although reasonably motivated, this choice is somewhat ad hoc. Again, though, we expect the broad behaviors of the spectrum to be correctly modelled; the results for the masses of the scalar, vector, and axial mesons are shown in Fig. 2 as a function of $N_{f}$. The value of $m_{V} / m_{A}$ at $N_{f}=2$ is input to constrain the model while the $N_{f}$ dependence is a prediction. Our main interest is in the large- $N_{f}$ region, and these results will be discussed in more detail below. 


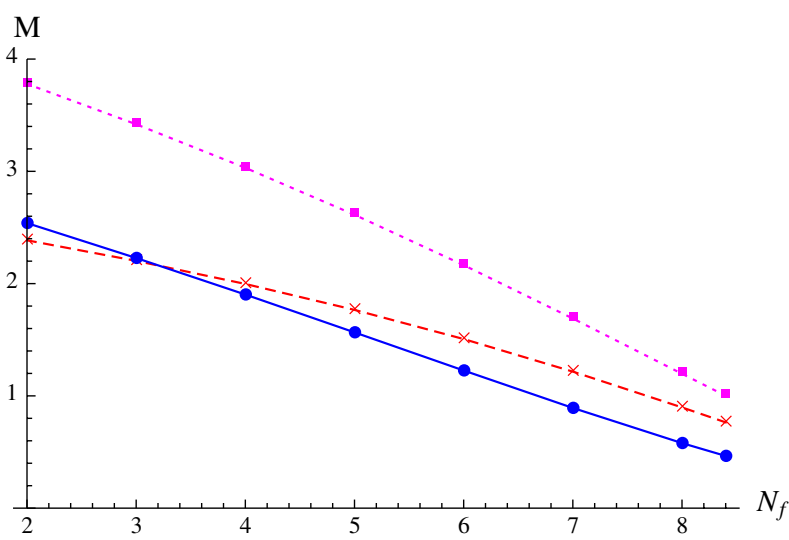

FIG. 2 (color online). Masses of the lightest scalar (dots), vector (crosses), and axial vector (squares) as a function of $N_{f}$. The values of $m_{v} / m_{A}$ at $N_{f}=2$ is input, and the $N_{f}$ dependence is a prediction of the model.

\section{A. Results at $\boldsymbol{m}_{q}=\mathbf{0}$}

The model is now completely fixed (there are no free parameters), and we can compute in the zero quark mass limit. First we solve Eq. (9) for the profile of the field $L$. The value of $L(0)$ is a measure of the dynamical quark mass in the IR, and we plot it against $N_{f}$ in Fig 3. Note that for $N_{f}<9.5$ the value of $L(0)$ saturates to a near constant. This seems reasonable because for all these theories, the scale at which the BF bound violation occurs is very similar, not separated by orders of magnitude of running (because of our initial condition on $\alpha$ ). One would expect very similar IR physics. Above $N_{f}=9.5$ the theories still have considerable running time between the fixed UV scale and the IR scale at which the BF bound is violated. For this reason $L(0)$ falls and shows considerable $N_{f}$ dependence. The scaling is of BKT type modulated by a power law in $N_{f}^{c}-N_{f}$,

$$
L(0)=a \exp \left(-b / \sqrt{N_{f}^{c}-N_{f}}\right)\left(N_{f}^{c}-N_{f}\right)^{p_{L}},
$$

where $a=4692.42, b=-5.111$, and $p_{L}=-0.722$. The numerical solution for $L(0)$ and the above fit are shown in Fig 3. Hence, we expect that the transition to the conformal window from the chiral symmetry breaking phase is continuous and displays Miransky scaling. Of course all dimensionful physical quantities in the theory are expected to show similar scaling. In practice, when fitted over a finite range in $N_{f}$, the scaling behavior of all quantities can be characterized by a BKT-type behavior, but with different fit parameters for different quantities. This suggests that while there is an IR scale which shows the expected BKT behavior and to which all other physical quantities are proportional, there can be additional $N_{f}$-dependent factors enhancing the scaling. This is precisely the behavior we have seen for $L(0)$ above.

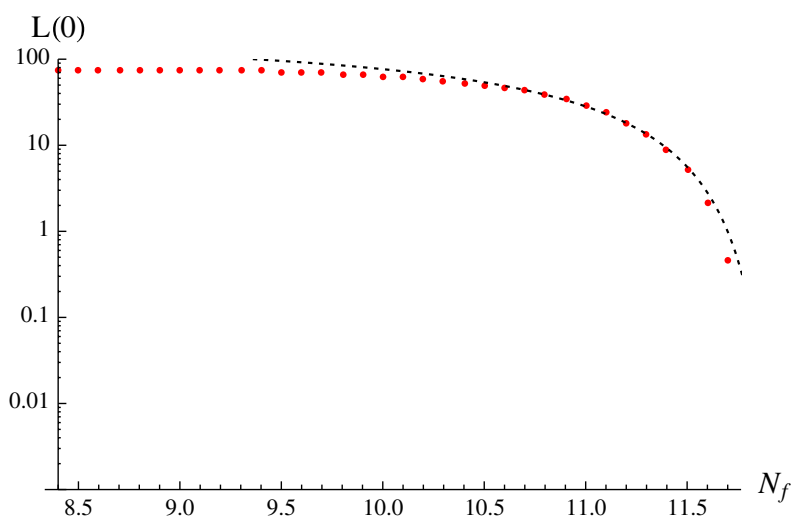

FIG. 3 (color online). The IR quark mass, L $(0)$ vs $N_{f}$. The dashed line shows the fit $a \exp \left[-b /\left(12-N_{f}\right)^{1 / 2}\right]\left(N_{f}^{c}-N_{f}\right)^{p_{L}}$, with $a=4692.42, b=5.111$, and $p_{L}=0.722$.

It turns out that there is a quantity, the quark condensate, which displays BKT scaling, i.e., a pure exponential form $a \exp \left(-3 b /\left(N_{f}^{c}-N_{f}\right)^{1 / 2}\right)$. In our model, we can determine the quark condensate from the asymptotic UV behavior of the profile $L(\rho)$ which is in the $m_{q}=0$ limit given by

$$
L(\rho)=\langle\bar{q} q\rangle \rho^{-2}(\ln \rho)^{k},
$$

where $k=3 C_{2} /\left(4 \pi b_{0}\right)$. The logarithmic correction is due to the running of the coupling. We show the numerical results for the condensate as well as the fit of the BKT form with parameters $a=63.090$ and $b=5.111$ in Fig. 4 .

The classic expectation of walking gauge theories is that theories, which have a large range over which $\gamma \simeq 1$, will have an enhanced UV quark condensate (the UV dimension 3 condensate is expected to be given by the product of the IR dimension 2 condensate and the UV scale at which $\gamma$ transitions to its higher value).

We plot the dimensionless $\langle\bar{q} q\rangle / f_{\pi}^{3}$ against $N_{f}$ in Fig. 5. When $N_{f}$ is sufficiently far below the conformal window,

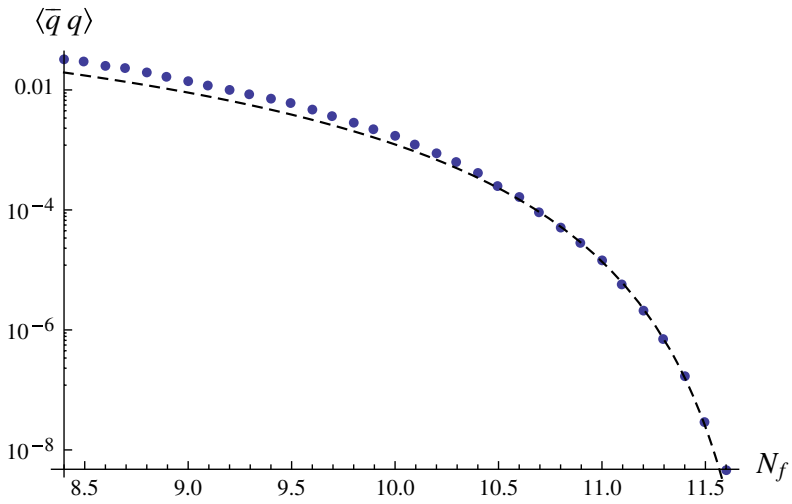

FIG. 4 (color online). The dots show numerical results for the quark condensate as a function of $N_{f}$. The dashed line is the BKT fit $a \exp \left(-3 b /\left(N_{f}^{c}-N_{f}\right)^{1 / 2}\right)$ with parameters $a=63.090$ and $b=5.111$. 


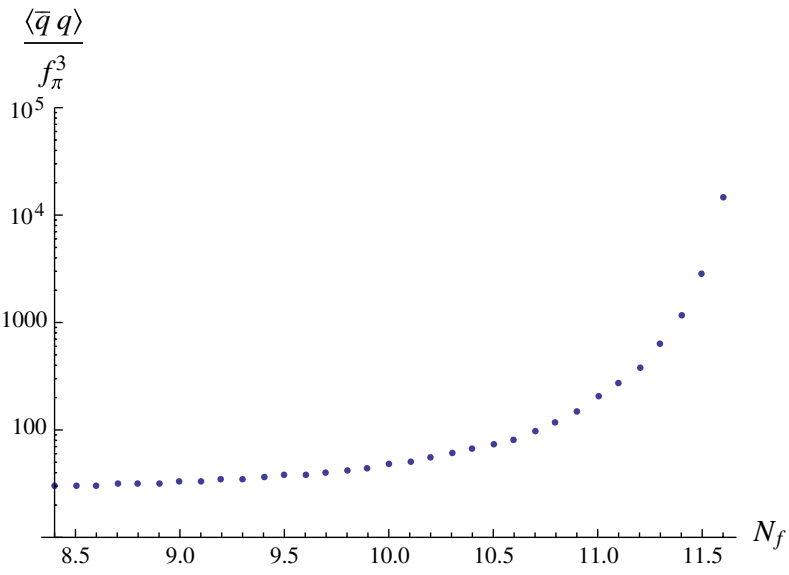

FIG. 5 (color online). The quark condensate normalized by $f_{\pi}^{3}$ vs $N_{f}$.

our results show the expected $\langle\bar{q} q\rangle \sim f_{\pi}^{3}$. However, when $N_{f}$ is increased, the condensate is enhanced relative to $f_{\pi}^{3}$. This enhancement of the condensate in the walking regime near the phase transition is a very clear prediction of the model.

We can now turn to the bound state masses and decay constants. As we have already discussed for the case of $L(0)$, we expect that the scaling of all physical scales near $N_{f}^{c}$ is

$$
O_{i}=A_{i}\left(N_{f}^{c}-N_{f}\right)^{p_{i}}\langle\bar{q} q\rangle^{1 / 3}
$$

where the overall normalization $A_{i}$ and the power $p_{i}$ are constants dependent only on the observable in question. Let us show this in detail for the masses of the scalar $(S)$, vector $(V)$, and axial $(A)$ mesons. We find that each of these quantities falls to zero according to Eq. (30) as shown in Fig. 6. In the figure the numerical results for the scalar masses are shown by dots, vector masses by crosses, and axial meson masses by squares. The dashed lines are the fits of the form (30) with $p_{S}=1.27, p_{V}=0.77$, and

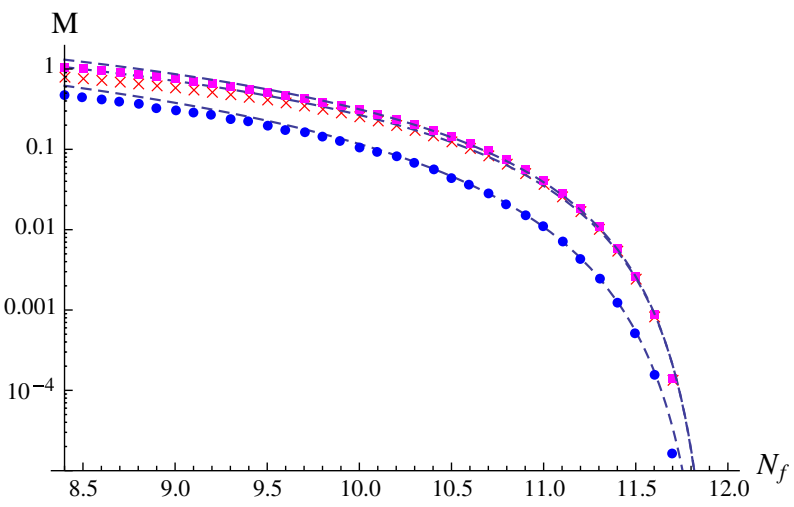

FIG. 6 (color online). Masses of scalar (dots), vector (crosses), and axial (squares) mesons as a function of $N_{f}$. The dashed lines show fits of the form $M_{i}=\left(N_{f}^{c}-N_{f}\right)^{p_{i}} L(0)$, where $p_{S}=1.27$, $p_{V}=0.77$, and $p_{A}=0.85$.
$p_{A}=0.85$. The fit for the pion decay constant is also of the same form and gives $p_{f_{\pi}}=1.02$.

Hence, we find that the masses scale toward zero faster than the pure BKT behavior given by the behavior of $\langle\bar{q} q\rangle$ alone. Among the masses the scalar mass behaves differently than the vector and axial meson masses. These results illustrate that one must be careful when extracting the scaling behaviors of various quantities with $N_{f}$ from lattice simulation results where only a finite range of discrete values of $N_{f}$ is accessible.

One of the phenomenologically most interesting questions is the ratio of these masses. We plot the ratio of the $V$ and $A$ masses to the scalar $(S)$ mass in the top plot of Fig. 7. It is clear that the scalar mass becomes light as one approaches the chiral symmetry transition at $N_{f}^{c} \simeq 12$. As in Ref. [9], our interpretation is that the potential for $\langle\bar{q} q\rangle$ becomes very flat as one approaches the transition-the large anomalous dimension of $\langle\bar{q} q\rangle$ over a wide running range leads to the enhancement of its vacuum expectation value as we have seen. The potential difference between the vacuum value and $\langle\bar{q} q\rangle=0$ is controlled by the IR, though, so it is order $f_{\pi}$. Thus, as one approaches the transition, the potential becomes arbitrarily flat, and a

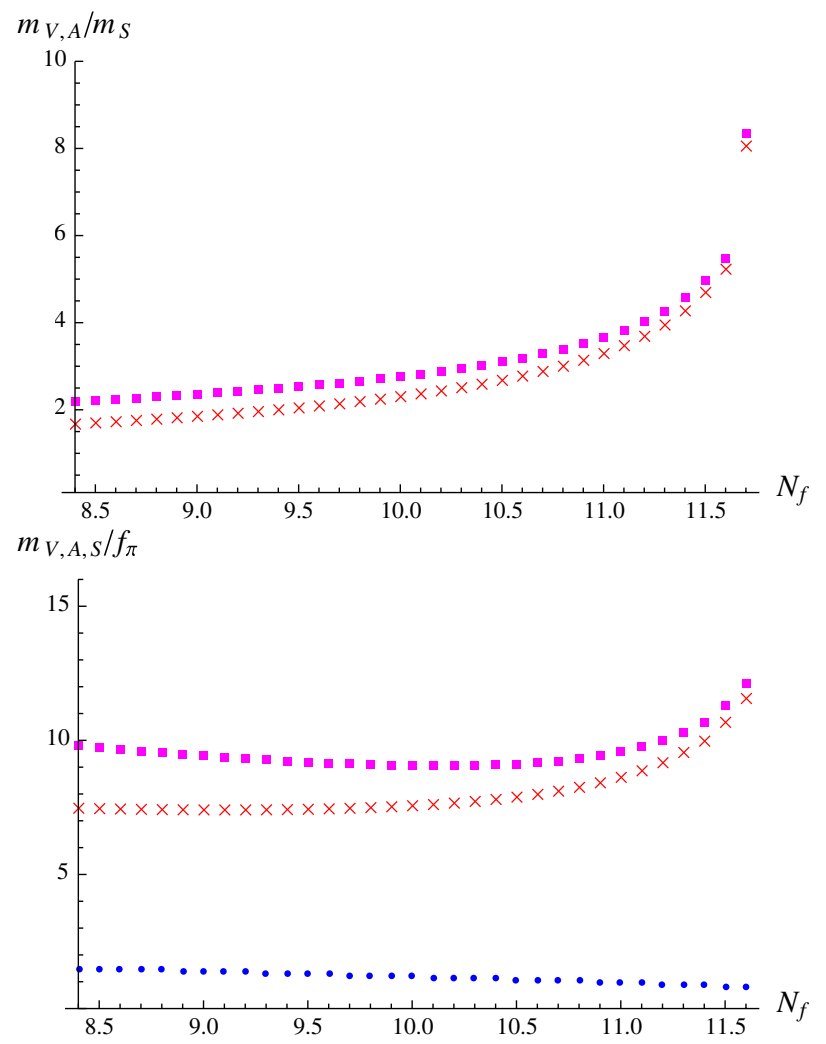

FIG. 7 (color online). Mass ratios: in the top figure the crosses and squares show the ratio of the $V$ and $A$ masses to the $S$ mass, respectively, revealing the Goldstone-like nature of the scalar meson as one approaches the critical point. The bottom figure shows the masses in units of $f_{\pi}$. The squares denote $m_{A}$, crosses $m_{V}$, and dots $m_{S}$. 
Goldstone associated with shift symmetry in energy emerges-the light $S$ meson. Note that in Ref. [9] we showed that the scalar became light relative to $L(0)$, but here in this extended model, we can see its Goldstone-like behavior relative to the $V$ and $A$ masses, $f_{\pi}$, and also the other decay constants as we will see below. The lightness of this state near the transition is rather clear in this model.

The lower plot in Fig. 7 shows the masses of the three mesons in units of $f_{\pi}$. As the transition point is approached, the $V$ and $A$ become degenerate by construction through our choice of the $N_{f}$ dependence in the parameter $\kappa$. At $N_{f}=2$ the ratio of the $V$ and $A$ masses matches that in QCD again by assumption.

The decay constants for the $V$ and $A$ in units of $f_{\pi}$ are shown in Fig. 8. Again by construction they become degenerate near the transition. In Fig. 9 we show the decay constant of the scalar meson divided by $f_{\pi}$. For $N_{f}$ sufficiently far below the conformal window, we observe that the obvious expectation of a QCD-like theory is satisfied as $f_{S} \sim f_{\pi}$. However, as $N_{f}$ is increased toward the boundary of the conformal window, we see a large hierarchy between $f_{S}$ and $f_{\pi}$ arising. This constitutes yet another clear prediction of our model in the walking region.

To conclude this analysis, we discuss the implications on electroweak physics. If one were to imagine using the gauge theories of the type we have analyzed here as technicolor models of electroweak symmetry breaking, then a key quantity to confront with existing electroweak data is the oblique corrections, in particular the $\mathrm{S}$ parameter [63]. The $\mathrm{S}$ parameter is given by

$$
S=4 \pi\left(\Pi_{A A}^{\prime}(0)-\Pi_{V V}^{\prime}(0)\right),
$$

where $\Pi$ are the vector vector and axial axial correlators. The derivative is with respect to $q^{2}$, and the derivatives are evaluated at $q^{2}=0$.

In the UV the correlators are given by Eq. (5), and since the axial symmetry is unbroken, the contribution to $\mathrm{S}$ is zero. Our insistence on the restoration of the vector axial

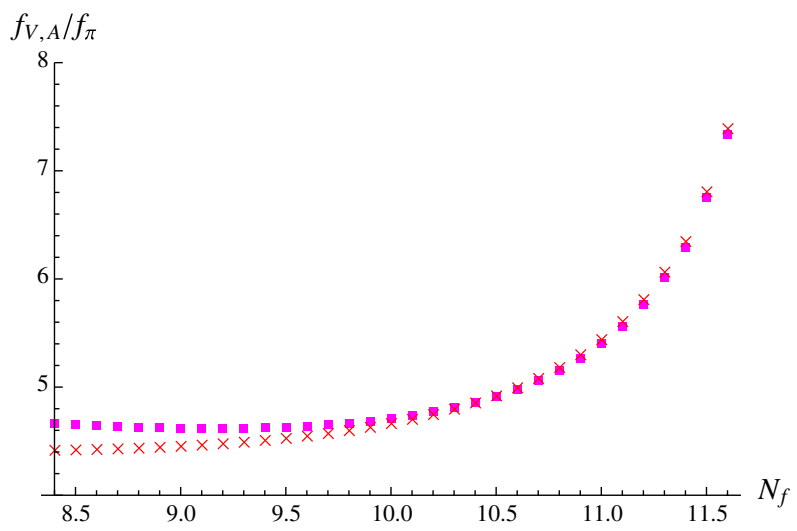

FIG. 8 (color online). The $V$ and $A$ decay constants, shown by crosses and squares, respectively, in units of $f_{\pi}$ against $N_{f}$.

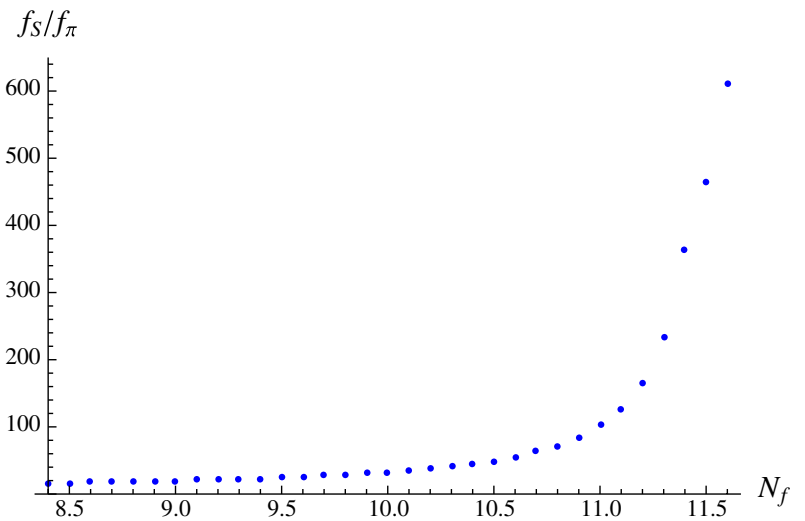

FIG. 9 (color online). Decay constant of the scalar meson divided by $f_{\pi}$ as a function of $N_{f}$.

symmetry in the spectrum as we approach $N_{f}^{c}$ will also mean that the $\mathrm{S}$ parameter vanishes at the BKT transition point.

$\mathrm{S}$ is usually computed in technicolor models through the contributions of the various bound states of the theory. For a model to be realistic in modelling the electroweak symmetry breaking of the Standard Model, the scalar meson must mimic the Standard Model Higgs with mass $125 \mathrm{GeV}$ (and appropriate couplings). Its loop contributions will, we assume, therefore be that of the Standard Model.

The $V$ and $A$ mesons will make a further contribution, which is of size

$$
S=4 \pi\left(\frac{f_{V}^{2}}{m_{V}^{2}}-\frac{f_{A}^{2}}{m_{A}^{2}}\right)
$$

We plot this extra contribution, normalized by the number of electroweak doublets, in Fig. 10. The behavior sensibly matches expectations: For QCD-like theories at lower $N_{f}$, the $\mathrm{S}$ contribution per doublet is larger (by an order 1 number) than the contribution of a mass degenerate perturbative doublet $(1 / 6 \pi)$. In the walking regime, the

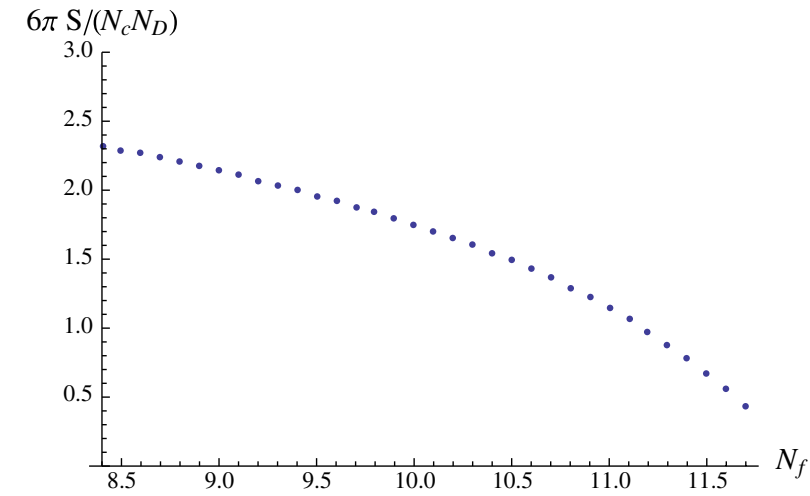

FIG. 10 (color online). The contribution to the $\mathrm{S}$ parameter (normalized by the number of technidoublets and to the perturbative value from a single mass degenerate doublet) from the lightest vector and axial mesons. 


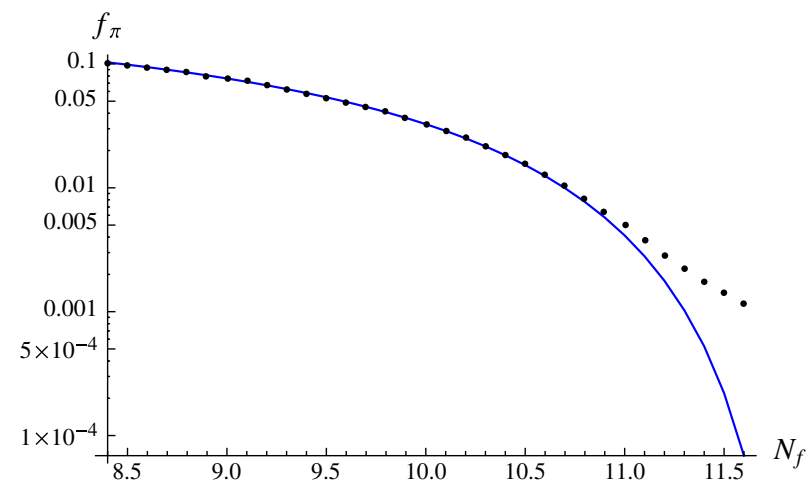

FIG. 11 (color online). The pion decay constant plotted against $N_{f}$. Solid line corresponds to $m_{q}=0$, while the dots correspond to $m_{q}=10^{-5}$.

contribution per doublet falls to zero at the chiral transition point.

\section{B. Results at $\boldsymbol{m}_{q} \neq \mathbf{0}$}

Finally let us investigate the effects of the small finite quark mass in the BKT region. In the massive case, the UV profile $L(\rho)$ has an additional non-normalizable piece [on top of the normalizable mode in Eq. (29)],

$$
L(\rho)=\frac{m}{(\ln \rho)^{k}},
$$

with $m$ interpreted as the quark mass. Note the dimension of the product $m \bar{q} q$ is always 4 in the model.

In Fig. 11 we show the pion decay constant for the cases $m_{q}=0$ and $m_{q}=10^{-5}$. We see that, while the finite quark mass curve traces well the corresponding zero mass curve at low $N_{f}$, it deviates as $N_{f}^{c} \simeq 12$ is approached. The transition corresponds to the point where the IR scale where the BF bound is violated becomes equal to the hard quark mass. Note that the bound states persist above

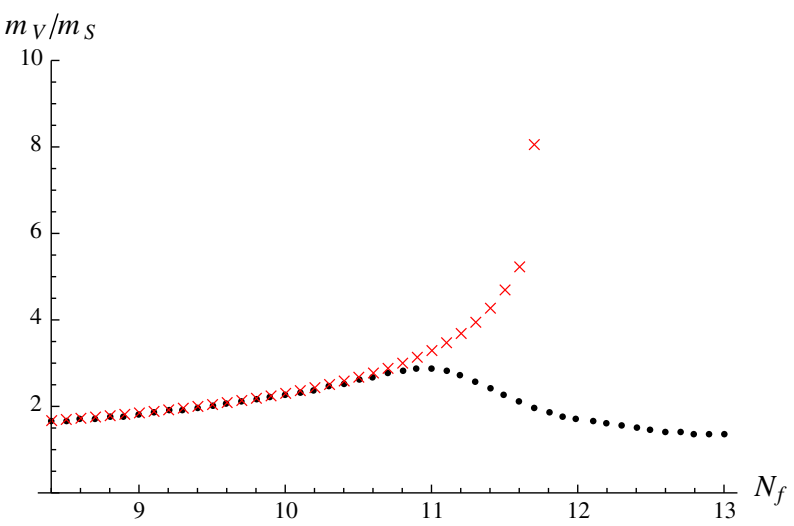

FIG. 12 (color online). The ratio of $m_{V} / m_{S}$ against $N_{f}$. The crosses show the vector meson mass in the $m q=0$ case while the black dots correspond to $m_{q}=10^{-5}$.

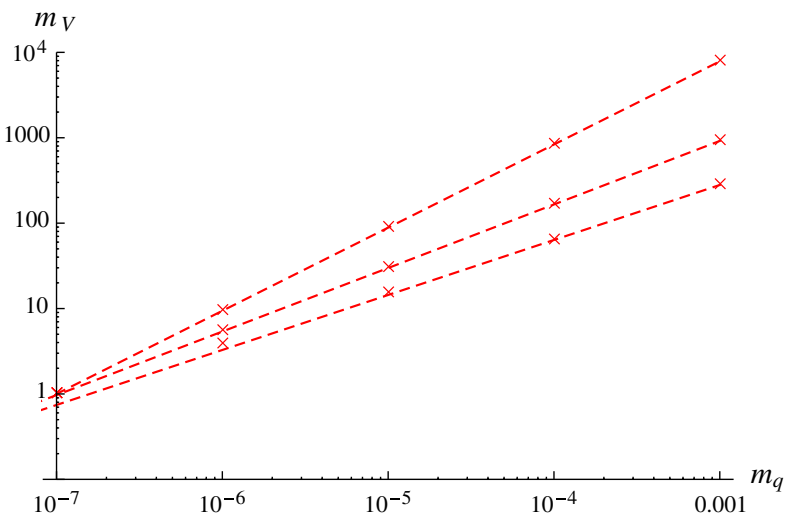

FIG. 13 (color online). $\quad m_{V}$ vs $m_{q}$ at $N_{f}=16,13,12$ from top to bottom. The dotted lines are fits of the form $m_{q}^{b}$ with $b=0.644$ for $N_{f}=12, b=0.744$ for $N_{f}=13$, and $b=0.974$ for $N_{f}=16$.

$N_{f}^{c}$ since the hard mass breaks conformality, and there is no conformal window.

The Goldstone nature of the scalar is also lost; Fig. 12 shows $m_{V} / m_{S}$ against $N_{f}$. Again, below $N_{f}=12$ the finite mass behavior coincides with the zero mass case, but as $N_{f} \simeq 12$ is approached, all states begin to scale with $m_{q}$ leading to the degeneracy implied by the figure.

To test the scaling behavior of the masses, we plot the $V$ mass against the current quark mass at $N_{f}=12,13,16$ in Fig. 13. They are well fitted by the curves $m_{q}^{b}$ with $b=$ $0.644\left(N_{f}=12\right), b=0.744 N_{f}=13$, and $b=0.974$ $N_{f}=16$. These powers are readily explained. For small enough quark mass, the quark mass has dimension $1+\gamma_{*}$, with $\gamma_{*}$ the IR fixed point value in the conformal regime. Hence, the dimension $1 \rho$ mass is expected to scale as $m_{q}^{1 /\left(1+\gamma_{*}\right)}$ [64] - this predicts $b=0.676, N_{f}=12, b=$ $0.770, N_{f}=13$, and $b=0.974, N_{f}=16$. The agreement is satisfyingly at the few percent level.

\section{DISCUSSION}

We have introduced a variant of AdS/QCD, which has a dynamical mechanism for the generation of the quark condensate and naturally introduces a soft wall at the scale of the quark condensate. The model is basically just the linearized form of the top-down D3/D7 model but with the running of the anomalous dimension of the quark condensate input by an ansatz through the AdS scalar mass. We have used the model to study the dynamics of SU(3) gauge theory with $N_{f}$ quarks (we keep $N_{f}$ as a continuous parameter in the parametrization of $\gamma$ ). The model is then completely fixed by its action (3), the imposition of the radially dependent mass using perturbative QCD results in Eq. (26), and the choice of the coupling $\kappa$ in Eq. (27). The latter is picked to match the ratio of vector and axial vector meson masses in $N_{f}=2 \mathrm{QCD}$ and to scale with $N_{f}$ so that 
the axial vector symmetry is restored at the continuous chiral transition at the edge of the conformal window.

We find it remarkable that such a basic model then rather simply reproduces the entire lore about walking technicolor dynamics. Chiral symmetry breaking is triggered by the anomalous dimension of the quark bilinear, $\gamma$, growing above 1 . The transition displays Miransky scaling (Fig. 3) at the chiral restoration transition. In the walking regime, the quark condensate grows relative to $f_{\pi}$ (Fig. 5), the scalar meson becomes light relative to the rest of the spectrum (Fig. 7), and the electroweak S parameter falls to zero (Fig. 10).

When a small current quark mass is introduced into the theory, the chiral transition is lost, and the spectrum moves at large $N_{f}$ to a scaling behavior where all dimensionful quantities scale as the appropriate power of $m_{q}$ (given its anomalous dimension at the IR fixed point). This provides an explicit model realizing the scalings proposed in Ref. [64] and should provide a very helpful guide for lattice simulations of these theories.

The model, of course, is simplistic, not least in using the perturbative running of the gauge coupling. For example, recent lattice simulations for $\mathrm{SU}(3)$ with $N_{f}=12$ have predicted IR fixed point values for $\gamma$ in the range of 0.386 [24], 0.459 [20], and 0.32 [22] to be compared with the one loop perturbative value 0.48 we use. Although the precise runnings are open to correction, the broad flavor of our results is likely to be correct assuming the fixed point behavior persists to sufficiently high values of $\gamma$ (the possibility of "jumping" [65] exists and would give very different results).

Our results are of intrinsic interest in the study of gauge theories with conformal windows, and we hope they will be of use in motivating lattice simulations of non-QCDlike strong dynamics. Finally, it remains a fascinating question as to whether such physics could still be compatible with the very constraining LHC data. If that is the case, technicolor dynamics remains appealing because it removes fundamental scalars from the standard model and allows the physics of flavor to live at scales that can potentially be probed experimentally. Walking does seem capable of hiding many of the deficiencies of technicolor dynamics. For example, our model suggests a rather rapid growth of the quark condensate if $N_{f}$ lies within $10 \%$ of its critical value. This would alleviate problems with flavor changing neutral currents and the $T$ parameter [66] from extended technicolor. In the same regime, the scalar meson becomes a light Higgs-like state with mass of order $f_{\pi}$ with as much as an order of magnitude gap between its mass and those of other bound states. In this regime the S parameter contribution of a doublet is also smaller than a perturbative doublet leaving more room in precision data. The tuning needed to achieve these results seem to be alleviated in these models because tuning to $N_{f}^{c}$ simultaneously tunes $\gamma \rightarrow 1$ and increases the running distance over which near conformality is present. Both features are phenomenologically helpful. A key challenge remains the rate of the scalar meson decay to two photons, which we have not addressed here. Of course, even if nature does not use these schemes, the study of the conformal window remains an important theoretical question.

\section{ACKNOWLEDGMENTS}

N.E. is grateful for the support of a STFC consolidated grant. T. A. thanks the Väisälä foundation for financial support. K. T. acknowledges support from the European Science Foundation (ESF) within the framework of the ESF activity entitled "Holographic Methods for Strongly Coupled Systems" and hospitality at University of Southampton during the time the research reported here was carried out.

\section{APPENDIX: D3/PROBE D7 SYSTEM}

The dynamics of the condensate formation and how it forms a soft wall in meson computations in dynamic AdS/QCD is based on the D3/probe-D7 model [3]. The D3-branes generate the duality between $\mathcal{N}=4$ super Yang-Mills theory and the space $\operatorname{Ad} S^{5} \times S^{5}$,

$$
\begin{aligned}
d s^{2} & =G_{M N} d x^{M} d x^{N} \\
& =\frac{r^{2}}{R^{2}} d x_{3+1}^{2}+\frac{R^{2}}{r^{2}}\left(d \rho^{2}+\rho^{3} d \Omega_{3}^{2}+d L^{2}+L^{2} d \phi^{2}\right),
\end{aligned}
$$

where $R$ is the AdS radius and $r^{2}=\rho^{2}+L^{2}$. To introduce $\mathcal{N}=2$ quark hypermultiplets into the gauge theory, probe D7-branes can be introduced in the $x_{3+1}, \rho, \Omega_{3}$ directions $\left(\xi^{a}\right)$. They then lie at fixed $\phi$ with a potentially nontrivial profile $L(\rho)$. That profile is determined by the Dirac Born Infeld probe action (in Einstein frame)

$$
S_{7}=T_{7} \int d^{8} \xi e^{\phi} \sqrt{P\left[G_{a b}\right]},
$$

where $\phi$ is the dilaton (dual to the gauge coupling). The pulled back metric seen by fields on the D7 world volume is

$$
P\left[G_{a b}\right]=G_{M N} \frac{d x^{M}}{d \xi^{a}} \frac{d x^{N}}{d \xi^{b}} .
$$

We have

$$
S_{7}=T_{7} \int d \rho e^{\phi} \rho^{3} \sqrt{1+\left(\partial_{\rho} L\right)^{2}+\frac{R^{4}}{r^{4}}\left(\partial_{x} L\right)^{2}} .
$$

In the supersymmetric case, the dilaton is constant, and the linearized equation of motion for the vacuum configuration $L(\rho)$ is just the first term in Eq. (9)-as is well known, the embedding profile encodes the quark mass and condensate holographically. The equation for the mesonic fluctuations about the vacuum embedding are then the 
first and last terms in Eq. (10). Note that it is crucial that the action has a prefactor of $\rho^{3}$ (not $r^{3}$ ) for the correct holographic relations to emerge. It is also crucial that in the embedding equation a factor of $r^{2}=\rho^{2}+L^{2}$ is present cutting off the space at $r$ of order the IR quark mass-if it were not there and the computation extended to $r=0$, the spectrum would become conformal. We have carried both of these aspects of the model across into our dynamic AdS/QCD model.

To understand how a radially dependent mass term for $L$ can emerge, we can consider a case with a nontrivial dilaton profile [8] and look at the action that controls the vacuum configuration of the D7. Note that the dilaton will naturally be a function of the radial coordinate of the background space, $r=\sqrt{\rho^{2}+L^{2}}$. Now if we linearize Eq. (A4) in $L$ and make the coordinate transformation

$$
e^{\phi} \rho^{3} \frac{d}{d \rho}=\bar{\rho}^{3} \frac{d}{d \bar{\rho}}
$$

we obtain the action

$$
S \sim \int d \bar{\rho} \bar{\rho}^{3}\left(\left(\partial_{\bar{\rho}} L\right)^{2}-\frac{\Delta m^{2}}{\bar{\rho}^{2}} L\right),
$$

with

$$
\Delta m^{2}=-\frac{\rho^{5}}{\bar{\rho}} e^{\phi} \frac{\partial e^{\phi}}{\partial \rho}
$$

The effect of a running coupling is precisely to introduce a radially dependent shift in the mass of the field $L$ as we have introduced in the dynamic AdS/QCD model in Eq. (7).
[1] J. Erlich, E. Katz, D. T. Son, and M. A. Stephanov, Phys. Rev. Lett. 95, 261602 (2005).

[2] L. Da Rold and A. Pomarol, Nucl. Phys. B721, 79 (2005).

[3] A. Karch and E. Katz, J. High Energy Phys. 06 (2002) 043; M. Grana and J. Polchinski, Phys. Rev. D 65, 126005 (2002); M. Bertolini, P. Di Vecchia, M. Frau, A. Lerda, and R. Marotta, Nucl. Phys. B621, 157 (2002); M. Kruczenski, D. Mateos, R. C. Myers, and D. J. Winters, J. High Energy Phys. 07 (2003) 049; J. Erdmenger, N. Evans, I. Kirsch, and E. Threlfall, Eur. Phys. J. A 35, 81 (2008).

[4] J. Babington, J. Erdmenger, N. J. Evans, Z. Guralnik, and I. Kirsch, Phys. Rev. D 69, 066007 (2004).

[5] J. M. Maldacena, Adv. Theor. Math. Phys. 2, 231 (1998); E. Witten, Adv. Theor. Math. Phys. 2, 253 (1998); S. S. Gubser, I. R. Klebanov, and A. M. Polyakov, Phys. Lett. B 428, 105 (1998).

[6] V.G. Filev, C. V. Johnson, R.C. Rashkov, and K.S. Viswanathan, J. High Energy Phys. 10 (2007) 019.

[7] N. Evans, A. Gebauer, M. Magou, and K.-Y. Kim, J. Phys. G 39, 054005 (2012).

[8] R. Alvares, N. Evans, and K.-Y. Kim, Phys. Rev. D 86, 026008 (2012).

[9] N. Evans and K. Tuomine, Phys. Rev. D 87, 086003 (2013).

[10] T. Appelquist, J. Terning, and L.C. R. Wijewardhana, Phys. Rev. Lett. 77, 1214 (1996).

[11] T. Appelquist, A. Ratnaweera, J. Terning, and L.C. R. Wijewardhana, Phys. Rev. D 58, 105017 (1998).

[12] T. Appelquist, K. D. Lane, and U. Mahanta, Phys. Rev. Lett. 61, 1553 (1988); A. G. Cohen and H. Georgi, Nucl. Phys. B314, 7 (1989).

[13] T. A. Ryttov and F. Sannino, Phys. Rev. D 78, 065001 (2008).

[14] T. A. Ryttov and F. Sannino, Phys. Rev. D 76, 105004 (2007).
[15] D. D. Dietrich and F. Sannino, Phys. Rev. D 75, 085018 (2007).

[16] F. Sannino and J. Schechter, Phys. Rev. D 60, 056004 (1999).

[17] A. Armoni, Nucl. Phys. B826, 328 (2010).

[18] H. Gies and J. Jaeckel, Eur. Phys. J. C 46, 433 (2006).

[19] Y. Aoki et al., Proc. Sci., LATTICE2011 (2011) 080.

[20] Y. Aoki, T. Aoyama, M. Kurachi, T. Maskawa, K. Nagai, H. Ohki, A. Shibata, K. Yamawaki, and T. Yamazaki, Phys. Rev. D 86, 054506 (2012).

[21] Y. Aoki, T. Aoyama, M. Kurachi, T. Maskawa, K.-i. Nagai, H. Ohki, E. Rinaldi, A. Shibata, K. Yamawaki, and T. Yamazaki, Phys. Rev. Lett. 111, 162001 (2013).

[22] A. Cheng, A. Hasenfratz, G. Petropoulos, and D. Schaich, J. High Energy Phys. 07 (2013) 061.

[23] A. Deuzeman, M.P. Lombardo, T. N. da Silva, and E. Pallante, Proc. Sci., LATTICE2011 (2011) 321.

[24] T. Appelquist, G. T. Fleming, M. F. Lin, E. T. Neil, and D. A. Schaich, Phys. Rev. D 84, 054501 (2011).

[25] A. Hasenfratz, Phys. Rev. D 82, 014506 (2010).

[26] Z. Fodor, K. Holland, J. Kuti, D. Nogradi, and C. Schroeder, Phys. Lett. B 681, 353 (2009).

[27] T. Appelquist, G. T. Fleming, and E. T. Neil, Phys. Rev. D 79, 076010 (2009).

[28] T. Appelquist, G. T. Fleming, and E. T. Neil, Phys. Rev. Lett. 100, 171607 (2008); 102, 149902(E) (2009).

[29] A. Deuzeman, M. P. Lombardo, and E. Pallante, Phys. Rev. D 82, 074503 (2010).

[30] Y. Shamir, B. Svetitsky, and T. DeGrand, Phys. Rev. D 78, 031502 (2008).

[31] Y. Iwasaki, K. Kanaya, S. Kaya, S. Sakai, and T. Yoshie, Phys. Rev. D 69, 014507 (2004).

[32] D. K. Hong and H.-U. Yee, Phys. Rev. D 74, 015011 (2006).

[33] D. Kutasov, J. Lin, and A. Parnachev, Nucl. Phys. B863, 361 (2012). 
[34] M. Jarvinen and E. Kiritsis, J. High Energy Phys. 03 (2012) 002.

[35] M. Jarvinen and F. Sannino, J. High Energy Phys. 05 (2010) 041.

[36] D. Arean, I. Iatrakis, and M. Jarvinen, Proc. Sci., Corfu2012 (2013) 129.

[37] O. Antipin and K. Tuominen, Mod. Phys. Lett. A 26, 2227 (2011).

[38] J. Alanen and K. Kajantie, Phys. Rev. D 81, 046003 (2010).

[39] J. Alanen, K. Kajantie, and K. Tuominen, Phys. Rev. D 82, 055024 (2010).

[40] D. Arean, I. Iatrakis, M. Jarvinen, and E. Kiritsis, Phys. Lett. B 720, 219 (2013).

[41] K. Haba, S. Matsuzaki, and K. Yamawaki, Phys. Rev. D 82, 055007 (2010)

[42] S. Matsuzaki and K. Yamawaki, Phys. Rev. D 86, 115004 (2012).

[43] D. Elander, C. Nunez, and M. Piai, Phys. Lett. B 686, 64 (2010).

[44] D. Elander and M. Piai, Nucl. Phys. B867, 779 (2013).

[45] D. Kutasov, J. Lin, and A. Parnachev, Nucl. Phys. B858, 155 (2012).

[46] M. Goykhman and A. Parnachev, Phys. Rev. D 87, 026007 (2013).

[47] W. E. Caswell, Phys. Rev. Lett. 33, 244 (1974).

[48] T. Banks and A. Zaks, Nucl. Phys. B196, 189 (1982).

[49] B. Holdom, Phys. Rev. D 24, 1441 (1981).

[50] S. Weinberg, Phys. Rev. D 13, 974 (1976).

[51] L. Susskind, Phys. Rev. D 20, 2619 (1979).
[52] R. Sundrum and S.D.H. Hsu, Nucl. Phys. B391, 127 (1993).

[53] T. Appelquist and F. Sannino, Phys. Rev. D 59, 067702 (1999).

[54] K. Yamawaki, M. Bando, and K. Matumoto, Phys. Rev. Lett. 56, 1335 (1986).

[55] M. Bando, K. Matumoto, and K. Yamawaki, Phys. Lett. B 178, 308 (1986).

[56] D. K. Hong, S. D. H. Hsu, and F. Sannino, Phys. Lett. B 597, 89 (2004).

[57] D. D. Dietrich, F. Sannino, and K. Tuominen, Phys. Rev. D 72, 055001 (2005).

[58] V. A. Miransky and K. Yamawaki, Phys. Rev. D 55, 5051 (1997); 56, 3768(E) (1997).

[59] D. B. Kaplan, J.-W. Lee, D. T. Son, and M. A. Stephanov, Phys. Rev. D 80, 125005 (2009).

[60] P. Breitenlohner and D.Z. Freedman, Ann. Phys. (N.Y.) 144, 249 (1982).

[61] G. Aad et al. (ATLAS Collaboration), Phys. Lett. B 716, 1 (2012).

[62] S. Chatrchyan et al. (CMS Collaboration), Phys. Lett. B 716, 30 (2012).

[63] M.E. Peskin and T. Takeuchi, Phys. Rev. D 46, 381 (1992).

[64] L. Del Debbio and R. Zwicky, Phys. Rev. D 82, 014502 (2010).

[65] F. Sannino, Mod. Phys. Lett. A 28, 1350127 (2013).

[66] R. S. Chivukula, B. A. Dobrescu, and J. Terning, Phys. Lett. B 353, 289 (1995). 